\title{
Labyrinthe
}

$35 \mid 2010$ (2)

Empire Reader

\section{La modernisation du colonialisme et les limites de l'empire}

\section{Frederick Cooper}

Traducteur : Pierre Savy

\section{OpenEdition}

Journals

Édition électronique

URL : http://journals.openedition.org/labyrinthe/4085

DOI : $10.4000 /$ labyrinthe.4085

ISSN : $1950-6031$

Éditeur

Hermann

Édition imprimée

Date de publication : 28 août 2010

Pagination : 69-86

ISBN : 9782705669973

Référence électronique

Frederick Cooper, "La modernisation du colonialisme et les limites de l'empire », Labyrinthe [En ligne], 35 | 2010 (2), mis en ligne le 27 juillet 2012, consulté le 19 avril 2019. URL : http://

journals.openedition.org/labyrinthe/4085; DOI : 10.4000/labyrinthe.4085

Propriété intellectuelle 


\title{
La modernisation du colonialisme et les limites de l'empire
}

\author{
Frederick COOPER \\ Contact : fred.cooper@nyu.edu
}

(C) 2006 Social Science Research Council. La version originale de ce texte est parue dans Craig Calhoun, Frederick Cooper, Kevin W. Moore (dir.), Lessons of Empire: Imperial Histories and American Power, New York, The New Press, 2006, p. 63-72. Reproduction sur autorisation. Version française revue et corrigée par l'auteur.

Il y a quelques années, les chercheurs les plus avancés proclamaient que l'État-nation se mourait, et que de nouveaux types de relations globales donnaient à l'ordre du monde une forme nouvelle. Voilà que l'État est de retour ; et, dans un cas, il l'est sous une forme si puissante que des intellectuels le qualifient d' «empire ». Des progressistes sérieux déploraient l'arrogance impériale du gouvernement américain ; des conservateurs pleins d'entrain ont alors contre-attaqué et
« Notre idée initiale était d'affirmer que l'impérialisme, tel que l'ont pratiqué surtout les pouvoirs européens du $x \mathrm{xII}^{\mathrm{e}}$ au $\mathrm{x} \mathrm{x}^{\mathrm{e}}$ siècle, n'est plus le modèle adéquat de la domination globale ; qu'en fait, une nouvelle forme de pouvoir global, de domination globale, était en train d'apparaître. Ainsi, dans l'impérialisme classique tout repose essentiellement sur les structures de la souveraineté nationale, autrement dit, sur l'imposition de celle-ci (qu'elle soit anglaise, française ou allemande, voire yankee, lorsque les ÉtatsUnis développèrent une version impérialiste de leur souveraineté) à des territoires étrangers, coloniaux mais aussi non-coloniaux ; tandis que la nouvelle idée de l'impérialisme, elle, ne repose pas sur l'État-nation. Nous entrons dans une ère où la souveraineté nationale ne peut suffire à des projets d'ampleur globale.

II leur faut en revanche - et c'est ce que nous entendons par "empire" - un réseau de pouvoirs qui fonctionnent en collaboration. Cela ne signifie pas que les États-nations n'ont plus aucune importance. Les États-nations sont importants, certains plus que d'autres, évidemment. L'empire auquel nous songions est un réseau de pouvoirs nationaux, mais aussi de pouvoirs économiques, d'organisations supranationales, voire d'organisations non gouvernementales. Cette structure de réseau décentré, c'est la forme d'ordre global qui se fait jour aujourd'hui. C'est autre chose que l'impérialisme tel que nous le concevions auparavant. » Michael Hardt, « Empire », Conversations with History, 12 mars 2004, URL : http://globetrotter.berkeley.edu/people4/Hardt/hardtcon6.html (visité le 19 mai 2010) 
affirmé que les États-Unis étaient bien un empire, dont le libre exercice d'un pouvoir étatique serait bon pour le monde tout entier ${ }^{1}$.

L'idée d'un dépérissement de l'État-nation était aussi fausse le 10 septembre 2001 qu'elle l'est aujourd'hui, et ce qui est particulièrement frappant - et, d'une certaine manière, lourd de présages — sur la façon dont les États-Unis projettent leur puissance, c'est qu'ils ne le font pas à la manière d'un empire. La relation entre des institutions territorialement limitées et des liens transterritoriaux a longtemps été évolutive, et la période de l'histoire que nous traversons actuellement ne fait pas exception à ce modèle. Mais l'observation de la manière dont cette relation évolue dans cette conjoncture n'est pas encouragée par des arguments qui, en affirmant la mort de l'État ou la domination d'un État, reviennent à dissoudre le problème.

La notion d'empire est utilisée comme une métaphore plutôt que comme une catégorie désignant une forme particulière de gouvernement. Mais certaines des questions cruciales que nous rencontrons portent sur les structures à travers lesquelles le pouvoir est exercé dans un ordre du monde asymétrique, sur les institutions

"Ce n'est probablement pas le bon terme, mais [Toni Negri et moi] n'avons tout simplement pas pu en trouver d'autre.

Harry Kreisler [animateur-producteur du programme "Conversations with History »] - Pour les ventes en librairie, cela a dû être un très bon terme.

- Possible, je ne sais pas. »

M. Hardt, « Empire », art. cit. qui peuvent conjuguer processus territoriaux et processus « déterritorialisés », sur les formes de souveraineté partagée qu'entraînent l'Union européenne et d'autres unités de coopération internationale, et sur le gouvernement d'entités politiques multiculturelles. Penser historiquement l'empire présente l'avantage de réduire l'État-nation à sa juste taille. Nous ne devons pas voir l'histoire comme une succession d'époques cohérentes — de l'empire à la nation, de la nation à la globalité post-nationale. En reconnaissant l'importance continue des empires jusqu'en plein $\mathrm{Xx}^{\mathrm{e}}$ siècle et l'importance de mouvements politiques qui cherchèrent à changer et à remplacer les empires, on en arrive à une question plus générale, dont l'importance ne s'est pas démentie : la gamme des possibilités d'exercice du pouvoir. Dans le passé comme dans le futur, des communautés peuvent être imaginées, mais toutes les imaginations ne sont pas nationales ${ }^{2}$. 
Un bon début, pour examiner les spécificités de ces formes politiques envahissantes, qui mettent à mal la souveraineté et sont porteuses de changement, est de commencer par distinguer entre l'impérial, l'hégémonique et le colonial $^{3}$ - quoique cette distinction en dise à la fois trop et pas assez : pas assez pour percevoir les vastes différences qui séparent ces registres en termes de processus et de conséquences, trop pour comprendre l'ambiguïté de ces registres et l'interaction à l'œuvre entre eux. Si l'on admet que la position américaine sur l'Irak ne renvoie pas à l'empire en un sens formel — qui passerait par l'affirmation d'une souveraineté durable sur l'Irak — et qu'elle est donc soit hégémonique, soit impériale, mais pas coloniale, on arrive toutefois bien vite à un problème qui, lui, est colonial. Prenez le catalogue suivant, tiré d'un éditorial publié récemment dans le New York Times : en Irak, les ÉtatsUnis ont devant eux

au moins une décennie de reconstruction et d'améliorations. Cela inclut la remise sur pied des ports, des fermes, des routes, des systèmes de télécommunications, des centrales électriques, des hôpitaux, du système de distribution de l'eau ; ainsi que l'introduction d'un système de sécurité sociale, d'un plan national pour les retraites, et de nouvelles lois pour les investissements étrangers et les droits de propriété intellectuelle. Le pays a besoin d'un code pénal et d'un système judiciaire révisés, d'une fiscalité nouvelle et d'un nouveau système de perception des impôts, et d'un système électoral doté d'une technologie appropriée... La facture totale s'élèvera sans doute à au moins 200 milliards de dollars sur une décennie ${ }^{4}$.

Cette description du travail à accomplir semble familière à l'historien des empires britannique et français en Afrique. Cela ne lui rappelle pas le colonialisme en général, mais celui d'après la Deuxième Guerre mondiale, quand les puissances coloniales majeures, essayant de rétablir leur légitimité, de remettre en ordre leurs empires et de les rendre plus productifs, reconnurent la médiocrité de leur contribution économique et sociale aux colonies et décidèrent d'une nouvelle politique de « développement ». Les buts du Colonial Development and Welfare Act britannique (adopté en 1940 et mis en œuvre en 1945) et du Fonds d'investissement pour le développement économique et social, créé par la France en 1946, sont fort similaires à ceux que l'on vient de mentionner. Et l'effort dura environ une décennie : au terme de celle-ci, gouvernements britannique et français, frustrés par les difficultés rencontrées dans ce projet si novateur, 
se rendirent compte que leurs contribuables ne consentiraient pas à payer la facture pour mener le projet à bien, que les interventions produiraient plus de conflits qu'elles n'en apaiseraient, et qu'il était plus prudent d'abandonner l'empire - dès lors que les buts en étaient si ambitieux - que d'essayer de le faire fonctionner.

Si l'on regarde plus loin en arrière, l'histoire des empires, désormais assez ample, va largement à rebours de l'usage polémique du concept d'empire : elle souligne que l'empire est une forme de domination limitée - limitée à la fois pour ce qui est de l'exercice d'un pouvoir totalisant et pour la volonté et la capacité de transformer les sociétés. Quand, plutôt tardivement, Grande-Bretagne et France se décidèrent sérieusement à utiliser la colonisation comme outil de transformation sociale, elles durent très vite reculer ${ }^{5}$. Je voudrais donc examiner maintenant de façon plus détaillée la notion des limites de l'empire — même dans des cas d'extrême asymétrie de puissance - et les contradictions de la modernisation du colonialisme.

\section{Les missions civilisatrices, les projets de développement, et leurs limites}

En évoquant une «mission civilisatrice », certains dirigeants de la Troisième République des années 1870 essayaient de convaincre leurs collègues et les électeurs que la conquête coloniale n'était pas nécessairement trop aventureuse pour une société bourgeoise, ni trop militariste pour une république. Les nouveaux sujets ne seraient ni pleinement incorporés, ni tenus dans un état d'altérité permanente. Cela correspondait aux notions plus anciennes de l'empire français comme gouvernement inclusif, mais différencié : le noyau se trouvait dans la France européenne ; les « vieilles colonies » dans les Caraïbes notamment, où, depuis 1848, tous les habitants étaient citoyens ; les avant-postes en Asie du Sud-Est et au Sénégal, où les habitants avaient certains droits civiques; quant à l'Algérie, qui était partie intégrante de la métropole, elle refusait la citoyenneté à la majorité de sa population. À la fin du XIX ${ }^{\mathrm{e}}$ siècle, la France acquérait de «nouvelles colonies », dont les peuples allaient être sujets mais non citoyens, et des protectorats, c'est-à-dire des États qui avaient cédé volontairement une partie de leur souveraineté à la France tout en conservant leur propre «nationalité ». Les colonisateurs républicains 
déclaraient que la France pouvait prendre en charge de nouveaux sujets à condition de s'engager à les « civiliser » et à leur donner la citoyenneté s'ils prouvaient leur valeur. Cette vision processuelle de la colonisation présumait que l'empire était une unité politique et morale. Bien plus tôt, la révolution haïtienne de 1791-1804 avait mis sur la table la question de l'application ou non de la Déclaration des droits de l'homme aux colonies, juste après la publication de celle-ci à Paris 6 .

Les Britanniques ne disposaient pas d'un tel édifice de la citoyenneté — tous étaient sujets de la reine ; mais le mouvement anti-esclavagiste eut un impact plus grand encore, en soulignant que la façon dont étaient traités les sujets de l'Empire, quelles que fussent leur couleur et leur culture, pouvait constituer une tache sur le drapeau britannique. Si, aux yeux de l'opinion britannique - ou de la partie de celle-ci qui s'en souciait - , la conquête entreprise à la fin du XIX siècle avait du sens, c'est en large part parce que, depuis les années 1860, un influent lobby missionnaire avait présenté l'Afrique comme un continent obscur, encore déchiré par le commerce intérieur d'esclaves, commandé par des tyrans, en mal de rédemption. Cependant, l'expérience de l'empire était déjà ambiguë : les espoirs de sauver les esclaves déjà britanniques des Indes occidentales avaient été anéantis par le refus de beaucoup de ces exesclaves de jouer le rôle de travailleurs respectueux et libres qui leur était assigné ; on voyait en eux, de plus en plus, des exceptions raciales à la règle de l'optimisation des comportements dans un marché libre et sous un juste gouvernement. Dans le discours impérial de la fin du XIX ${ }^{\mathrm{e}}$ siècle, le discours de la rédemption et celui de l'infériorité inéluctable coexistaient difficilement ${ }^{7}$. Et, à côté de cette ambivalence, des fonctionnaires interprétaient diversement les risques que présentait une attitude trop dure : la mutinerie indienne de 1857 mena le gouvernement à exiger que l'Inde fût gouvernée fermement, mais soigneusement.

Les empires étaient des gouvernements à la fois intégrateurs et différenciés. Que la différenciation soit trop vive, et les autorités intermédiaires dont dépendait le centre n'auraient pas d'intérêt à la coopération; que l'incorporation soit trop forte, et la revendication d'une expression ou d'une autonomie politiques serait permise. En France et en GrandeBretagne, certains groupes de pression concevaient l'espace de l'empire en termes moraux ; ils soutenaient que l'esclavage ou l'abus de populations sujettes violaient l'intégrité impériale. Cela n'empêchait pas chez 
les gouvernements impériaux et les colons les comportements brutaux et exploiteurs, mais cela assurait que le scandale serait un trait périodique du gouvernement impérial dans les États démocratiques — scandale à propos de l'esclavage, des massacres, des guerres coloniales, du travail forcé et de la pauvreté.

Les actions politiques de ceux qui voulaient réformer l'empire ne rencontraient que difficilement les mouvements politiques au sein des populations colonisées ellesmêmes - mais ces mouvements ne se limitaient pas à l'alternative binaire entre demander la pleine participation à la politique impériale dans les termes de cette politique ou bien transformer la différence en autonomie nationale. Des formes différentes d'affirmation culturelle et de revendication d'une expression et d'une représentation politiques — transformer les colonies en départements français, transformer l'empire en une fédéra-

Rome. Un débat au Sénat : faut-il donner la citoyenneté romaine aux barbares ou les réduire en esclavage?

"Le porte-parole de César Commode - Un jour César me demanda : à quel moment Rome fut-elle plus grande et plus forte ? Et je répondis à César : jamais Rome ne fut plus grande et plus forte que maintenant. Et à quoi devons-nous que l'empire soit ce qu'il est aujourd'hui ? À notre dureté, à notre intransigeance. Égalité, liberté, paix... qui donc fait usage de ces mots à part les Grecs, les juifs et les esclaves ? Rappelons-nous que derrière ces barbares il y en a des milliers d'autres, dont les hordes guettent une défaillance de notre part pour nous anéantir. Si nous acceptons ces barbares parmi nous, nos ennemis se hâteront de proclamer que nous sommes faibles; alors ils fondront sur nous de toute part et ce sera la fin de l'empire romain. Ce sera la fin de Rome !

Le sénateur Caecina [Finlay Currie] — La fin de Rome ? (Un temps) Comment disparaît un empire ? Est-ce qu'il s'effondre d'un seul coup, dans un tremblement de terre ? Non... non! Mais il arrive un temps où les peuples cessent peu à peu d'y croire et c'est alors qu'un empire commence à chanceler. Patriciens ! J'ai vécu sous quatre grands empereurs : Trajan, Hadrien, Antonin, Marc-Aurèle, et au cours de tant d'années notre empire a grandi, a changé ; la loi de la vie est de grandir ou mourir ! Et vous, sénateurs, vous êtes le cœur de Rome, c'est par votre voix que le peuple s'exprime. Parlez pour que le monde vous entende! Que le monde sache que Rome ne mourra pas. Ils sont des millions, des millions qui guettent à nos frontières. Si nous ne leur ouvrons pas nos portes, ils les briseront, et nous briseront nous aussi. Mais au lieu de cela, cherchons à grandir de plus en plus et à nous étendre ! Accueillons ces gens parmi nous! C'est avec leur aide que l'empire grandira encore. Honorables patriciens, nous avons changé le monde, ne pourrons-nous pas nous changer nous-mêmes ? » Anthony Mann, La Chute de l'Empire romain (Paramount Pictures, 1964). 
tion de nationalités distinctes, et d'autres formes de connexion transnationale (panislamisme, panafricanisme) - faisaient partie de la mobilisation politique dans et contre les empires. Jusqu'à la fin de la domination coloniale, l'intersection entre ces différentes sortes de mouvements soulignait l'importance de l'empire comme unité dans laquelle la moralité politique, les droits et le bien-être étaient débattus et marquaient l'instabilité de l'équilibre entre inclusion et différenciation. Que l'empire, dans les deux derniers siècles, ait provoqué dans les métropoles impériales un débat, s'organisant en réseaux internationaux et par l'opposition et la mobilisation dans les colonies, faisant usage des idéologies des puissances impériales elles-mêmes et les mettant en question, c'est un point qui contribue à expliquer l'inquiétude actuelle sur les conséquences politiques de la façon dont les États-Unis (ou une autre puissance) agissent quand ils interviennent fortement hors de leurs frontières ${ }^{8}$.

Il était plus compliqué de diriger un empire que ne le reconnaissaient les civilisateurs, les réformateurs et les rédempteurs. Le grand avantage dont devait disposer une puissance conquérante, à l'époque du télégraphe et de la mitrailleuse Maxim, c'était la capacité de concentrer des forces et de les déplacer. « Coloniser » consistait en ceci : fondre sur un village, terroriser de façon visible sa population, prendre le bétail, brûler les huttes, et puis s'en aller. Rendre le contrôle routinier était une autre histoire, qui supposait d'aller contre la logique de toute mission civilisatrice ou de tout projet d'édification d'un ordre politique nouveau. La seule façon d'administrer les larges espaces et les populations dispersées d'Afrique était de coopter des membres des élites locales auxquels étaient confiées les basses besognes. L' «indirect rule » était en Afrique une réalité - comme il l'avait été dans bien d'autres empires - longtemps avant de devenir une doctrine. En outre, France et Grande-Bretagne considéraient comme sacrée une ancienne doctrine impériale selon laquelle les colonies devraient payer pour leurs propres besoins. Même le célèbre avocat impérial Sir John Seeley déclara à propos de l'Inde, dans les années 1880 : «C'est une condition de notre Empire indien qu'il doive être tenu sans grand effort. » Le colonialisme britannique et français, au moins jusqu'à la Seconde Guerre mondiale, était un colonialisme à moindres frais : les colonies étaient censées payer les coûts de leur propre répression'.

Niall Ferguson, dans le corps de son livre Empire, établit que la domination britannique était limitée en raison de son faible coût et de sa 
dépendance à l'égard des autorités intermédiaires ; puis, à la fin du livre, il se contredit, en insistant sur le fait que la domination britannique signifiait autorité de la loi, gouvernement équitable et honnête, maintien de la paix et politique du libre marché. Elle n'avait ni la volonté ni la capacité de jouer un tel rôle, et certainement pas en Afrique, où la domination sur une bonne part du continent dépendait du renforcement du pouvoir des autorités locales, de l'usage d'une loi « coutumière » qui était réifiée en même temps que codifiée, de l'établissement de colons sur la base de l'accaparement de terre aux dépens des Africains et de l'interdiction de la propriété foncière africaine ailleurs, et sur la ségrégation des services publics. La politique économique favorisait les entreprises commerciales britanniques au détriment des réseaux commerciaux indigènes, et, comme Ferguson l'admet, elle invoquait la «préférence impériale » pour restreindre le commerce au-delà de la zone sterling quand cela correspondait aux besoins britanniques. $\mathrm{Si}$, aux colonies « blanches », l'Empire britannique donnait des leçons de droit et de libre marché, il prodiguait en revanche un enseignement tout différent aux Africains ${ }^{10}$.

Parfois, dans les premières années de la colonisation africaine, des fonctionnaires cherchèrent à transformer les cultivateurs ou les esclaves africains en travailleurs salariés ou en fermiers productifs. Le zèle consacré à ces efforts eut tôt fait de s'épuiser, en partie parce que les autorités dont dépendaient les dirigeants avaient sans doute intérêt à maintenir les peuples dans un état de dépendance (même si, formellement, l'esclavage avait pris fin), en partie parce que le besoin d'exporter les récoltes était trop fort pour que les autorités posassent trop de questions sur la façon dont elles étaient produites, et en partie parce que de nouveaux acteurs, notamment des colons blancs, poussaient à l'aménagement des principes du travail « libre». Tacitement, les fonctionnaires — parfois contre les protestations des missionnaires - $s$ 'accommodèrent d'une variété de formes d'organisation du travail dans les territoires africains. Après la Première Guerre mondiale, ils se félicitèrent d'avoir eu la sagesse de ne pas trop changer la société africaine.

Au début des années 1920, les gouvernements français et britannique rejetèrent les programmes de « développement » prévoyant le recours aux fonds métropolitains pour favoriser un usage plus systématique des ressources africaines, à la fois au nom de la vieille doctrine selon laquelle chaque colonie doit payer son propre développement et parce que des figures-clefs ne voulaient pas que les arrangements tacites avec les autorités 
africaines locales fussent bouleversés ${ }^{11}$. L'investissement de capital privé était un peu plus agressif ; les exportations de capital britannique allaient aux anciennes colonies et aux dominions, à l'Europe et aux États-Unis, et le peu qui allait à l'Afrique se dirigeait irrésistiblement vers les mines ${ }^{12}$. Durant toute cette période, on mit sur pied des enclaves exportatrices : les zones minières, entourées de vastes régions appauvries où l'on pouvait recruter des travailleurs pendulaires; des régions peuplées de blancs où des fermiers recevaient une aide considérable de l'État pour le recrutement et l'encadrement du travail; et des régions de culture où des fermiers africains, à petite ou moyenne échelle, avaient recours au travail familial, à des cultivateurs et des clients, et parfois au travail salarié. Les tentatives d'édifier un capitalisme indigène ou de colons se heurtèrent au fait que la plupart des Africains possédaient des ressources foncières, même si elles étaient réduites par l'aliénation ; en outre, les économies coloniales ouvraient de nouvelles niches, permettant d'échapper à la soumission à un employeur ; et les employeurs eux-mêmes ne voulaient pas nécessairement des règles d'une économie de travail salarié ${ }^{13}$. Les infrastructures se concentrèrent sur les voies étroites d'une économie commerciale. Les centres urbains offraient peu d'infrastructures pour les travailleurs à long terme et leurs familles. Une telle structure économique permit à certaines entreprises de réaliser de grands profits, mais elle encouragea les Africains à trouver des alternatives à une pleine implication dans le travail salarié ou l'agriculture commerciale.

\section{Vers le colonialisme de développement — et vers la fin de l'empire}

La médiocrité de la performance économique coloniale permit aux puissances coloniales de se débarrasser dans le monde rural, qu'elles n'avaient guère besoin de connaître, des dommages causés par la crise de 1929. À la fin de la décennie, toutefois, des fonctionnaires britanniques commencèrent à reconnaître que même les économies faibles d'Afrique et des Indes occidentales produisaient du désordre social, particulièrement dans les voies étroites de communication et dans les « îlots » de production salariée. Quand la production augmenta de façon hésitante (et non sans inflation), une vague de grèves commença, de la Barbade à Mombasa. Le Colonial Office, après une série de commissions et de débats internes, finit par reconnaître l'inadaptation déplorable des infrastructures et la 
pauvreté des colonies, à la fois anciennes (Indes occidentales) et nouvelles (Afrique). Avec l'adoption du Colonial Development and Welfare Act en 1940, on commença enfin à investir des fonds métropolitains, dans l'intention spécifique de renforcer les économies coloniales pour un développement de longue haleine et d'améliorer le bien-être au moins de ceux des sujets coloniaux qui se trouvaient dans le secteur « moderne ». Ce fut alors seulement que les initiatives fragmentaires et insuffisantes des gouvernements coloniaux en matière de santé, de techniques agricoles et d'urbanisme furent agrégées dans la catégorie du « développement ».

Durant le bref Front populaire (1936-1938), le gouvernement français entreprit lui aussi une autocritique de ses initiatives en Afrique. On les jugea insuffisantes : trop de contrainte, pas assez de raisons données aux Africains de participer volontairement à la production exportatrice, des infrastructures trop faibles. Mais, encore une fois, le corps législatif refusa de dépenser de l'argent en programmes de développement. Situé à droite politiquement, le gouvernement de Vichy, au début des années 1940, mit sur pied un plan pour une exploitation tranquille et systématique de l'Afrique, mais sans argent pour le mettre en pratique. La guerre, la vague de grèves - qui frappa l'Afrique française sitôt après la guerre - et d'autres conflits dans les colonies africaines, le tumulte dans d'autres colonies, notamment le Vietnam et l'Afrique du Nord, et l'environnement international changé poussèrent le gouvernement français, comme le gouvernement britannique, à consentir à des efforts pour rendre le colonialisme progressiste, d'où le mouvement de développement lancé après-guerre et les efforts variés de réforme politique. À la différence de la catégorie plus ancienne de "civilisation», le « développement» renvoie d'abord à des changements concrets et à un progrès mesurable. Cette catégorie, qui établit une hiérarchie entre ceux qui ont atteint le développement et ceux qui ne l'ont pas atteint, constitue aussi un langage dans lequel on peut revendiquer les ressources permettant d'avancer dans ce sens.

Mais le cadre du développement ne mettait pas la question sociale sous contrôle. La main-d'œuvre était une force rétive, que les fonctionnaires britanniques et français essayaient de domestiquer en important en Afrique les techniques des relations industrielles. La campagne africaine étant vaste et trop résistante aux changements, il fallait couper de leurs origines rurales les travailleurs salariés africains, pour définir, former et acculturer une force de travail africaine dans les sites-clefs de 
la production et du commerce, dans les mines et les villes et le long des voies ferrées : cette politique est connue sous le nom de « stabilisation ». Il s'agissait de verser des salaires adaptés pour faire vivre une famille et faire croître la génération suivante de travailleurs sous la surveillance d'experts en nutrition, en éducation et en relations professionnelles. Les syndicats se rendaient compte de la vulnérabilité des régimes coloniaux à l'action collective dans les voies étroites d'une économie coloniale, et de la vulnérabilité des efforts idéologiques aux manifestations de mécontentement. Dès lors que les régimes coloniaux n'étaient plus capables de défendre une frontière racialement définie, il était difficile de mettre de côté les revendications à « un même salaire pour un même travail »— revendications posées dans le langage même du colonialisme de développement.

Le cas français offre une démonstration particulièrement vivante de la volatilité d'une politique supranationale où l'effort de la métropole pour se définir comme le modèle et la référence en matière de progrès mène, de façon non intentionnelle, à une escalade des revendications exposées dans le langage de l'idéologie impériale. En 1946, le corps législatif français, qui comptait alors une petite minorité de représentants coloniaux, adopta une loi qui abolissait la distinction entre sujet et citoyen. La loi de la citoyenneté entendait peut-être donner aux Africains une voix minoritaire dans les institutions françaises tout en leur refusant le contrôle sur leur propre destinée - et en affirmant dans le même temps le caractère indissoluble et unitaire de la Grande France ; mais l'effet obtenu fut plus profond que cela. Une fois l'idéologie impériale dépouillée de l'idée d'une normalité de la distinction raciale, la notion d'empire comme unité politique et morale devint une force pour les mouvements sociaux. Les syndicats revendiquaient un salaire égal pour un travail égal ; les vétérans revendiquaient des pensions complètes ; les étudiants refusaient que leur éducation demeurât inférieure ; les partis politiques demandaient plus de dépenses de santé et d'aménagements urbains — tous s'exprimaient dans la rhétorique de la citoyenneté française.

Ces exigences étaient soulignées par des grèves et d'autres formes d'action collective, mais elles en appelaient aussi aux administrateurs, dont les espoirs pour modifier l'empire supposaient que les Africains prissent au sérieux les prémisses de la modernisation de l'impérialisme. Les mouvements politiques africains, tout en revendiquant toujours plus d'autonomie, se servaient pleinement des institutions impériales et de la rhétorique de la 
« Un ami et collègue m'a dit que le premier devoir d'un intellectuel yankee est d'être anti-américain. Eh bien, je ne suis pas d'accord. Non que l'on doive pardonner toutes les horreurs commises par le gouvernement yankee, je ne veux pas dire cela du tout. Mais si l'on doit reconnaitre celles-ci, on doit dans le même temps récupérer les grands courants de l'histoire yankee (mais aussi française, mais aussi anglaise) qui constituent en fait une base pour les luttes à venir. »

M. Hardt, « Empire », art. cit. citoyenneté pour forger leurs revendications. En 1956, un ministre français résuma le tour qu'avait récemment pris la politique impériale : la citoyenneté avait fini par signifier «l'égalité dans les salaires, l'égalité dans la législation du travail, dans le bénéfice de la sécurité sociale, l'égalité dans les allocations familiales, en bref, l'égalité des niveaux de vie ${ }^{14} »$.

Ainsi n'est-ce que dans la dernière phase de la domination coloniale que quelque chose comme le projet d'un impérialisme réformateur fut mis en pratique avec un minimum de sérieux. Et cet effort eut la vie courte. Le problème fondamental du colonialisme de développement avait deux faces, l'une et l'autre lourdes de sens si l'on considère le temps présent. Un problème était d'ordre intérieur : les empires français et britannique étaient des empires à moindres frais. Le colonialisme d'après-guerre promettait de coûter cher ${ }^{15}$, et cette promesse fut rapidement tenue. L'effort de développement s'enlisa dans les infrastructures rétrogrades des colonies africaines, alors que les travailleurs africains étaient en bonne position pour revendiquer de plus hauts salaires et des bénéfices, ce qui eut pour conséquence d'augmenter les coûts. Les archives coloniales trahissent la frustration des fonctionnaires du début des années 1950 face à la lenteur des résultats obtenus sur le front du développement. À l'intérieur, l'empire ne faisait plus recette, et, au milieu des années 1950, en France et en Grande-Bretagne, la presse et le corps législatif s'interrogeaient sur son coût ${ }^{16}$.

Le deuxième problème se trouvait en Afrique même. L'effort de réforme produisait plus de conflits qu'il n'en apaisait. Les syndicats prirent confiance, leurs exigences grandirent. Les fermiers qui réussissaient le mieux se plaignirent de la discrimination sur les marchés, et ceux qui ne réussissaient pas se plaignirent — et parfois se révoltèrent — en raison de leur situation d'insécurité accrue dans un environnement plus compétitif ${ }^{17}$. Tout cela s'ensuivit quand la logique de l'empire rencontra la logique de l'État-providence, tandis que la logique de la distinction raciale immuable n'était plus tenable. L'État devint responsable de 
l'empire tout entier, et les organisations sociales et politiques à travers l'empire étaient prêtes à poser des problèmes d'équivalence et d'égalité dans l'espace impérial.

À la fin, ce furent les gouvernements français et britannique qui vacillèrent : ils ne pouvaient accepter les coûts de la modernisation du colonialisme quand les bénéfices politiques et économiques étaient si incertains. Dans l'Afrique française, les Africains hésitaient encore entre la revendication de plus d'autonomie et celle d'une citoyenneté française plus complète, quand le

« En tant qu'assomption de l'impossibilité de l'État moderne, l'Empire est aussi bien l'assomption de l'impossibilité de l'impérialisme. La décolonisation aura été un moment important de l'établissement de l'Empire, logiquement marqué par la prolifération d'États fantoches. La décolonisation signifie ceci de nouvelles formes de pouvoir horizontales, infrainstitutionnelles, ont été élaborées qui fonctionnent mieux que les anciennes ».

Tiqqun, Contributions à la guerre en cours, Paris, La Fabrique, 2009 [2001], p. 61. gouvernement français décida de confier l'essentiel du pouvoir, y compris le budget, aux différents territoires coloniaux. L'unité dans laquelle les citoyens chercheraient l'égalité — et les ressources pour la payer — serait le territoire, non l'empire. Pour la France, comme pour la Grande-Bretagne, cela revenait à renoncer vraiment au pouvoir de contrôle impérial, mais, pour les Africains qui conclurent le marché, cela revenait à renoncer aux revendications sur ces ressources en échange d'un pouvoir territorialement limité.

C'est le colonialisme le plus réformateur qui s'effondra le premier en Afrique. Et la colonisation portugaise, la plus ancienne et la moins dynamique des colonisations africaines, fut la dernière à disparaître. Il est important de penser au-delà de la notion commune d'un édifice colonial obstiné et immuable qu' auraient fait tomber d'héroïques soulèvements. Les soulèvements en Afrique subsaharienne, notamment à Madagascar, au Kenya et au Cameroun, furent réprimés avec succès - et de même, pour ce qui est des aspects militaires, en Algérie ${ }^{18}$. Mais la menace venait aussi de l'action collective à l'intérieur de l'édifice colonial ; elle venait d'Africains qui retournaient les arguments modernisateurs de la GrandeBretagne et de la France en revendications de richesses, et dont les grèves, les manifestations et autres campagnes - dans l'ombre de l'Algérie, de Suez, du Vietnam et autres crises coloniales - obligèrent la GrandeBretagne et la France à faire à propos des colonies le calcul des coûts et des bénéfices ; un calcul qu'elles n'avaient jamais dû faire avant. 


\section{Implications}

Que signifie cette histoire pour la conjoncture du monde contemporain ? Elle révèle des modèles alternatifs d'empire. L'idée d'empire comme mécanisme transformateur est tenable, mais on doit faire attention à la façon dont on la situe. Les précédents ne sont pas l' «Empire britannique » ou la mission civilisatrice française au sens fort, mais plutôt les initiatives pour refaire systématiquement apparaître les sociétés colonisées comme une alternative dans les régimes coloniaux, contre d'autres visions de la colonisation, de l'origine impitoyable et déshumanisante à la conservation délibérée de communautés indigènes pacifiées, avec, dans tout cela, une bonne dose d'improvisation à bas coût. Le précédent concernant l'impérialisme interventionniste paraît assez adapté aux préoccupations d'aujourd'hui, mais pas dans le sens où les avocats du modèle de l'empire l'entendraient : c'est un précédent pour en sortir quand les coûts deviennent élevés. Si ceux qui défendent la transmission du manteau impérial de la Grande-Bretagne aux États-Unis se demandent si les Américains veulent se charger d'une mission impériale, l'expérience britannique devrait en fait renforcer leur anxiété.

L'autre modèle de colonialisme qui semble pertinent est ce que Ferguson (mais nous ne parlons pas des passages où il

" L'Empire empire » : titre de une de Libération, 4 novembre 2004, après la réélection de George W. Bush.

tire des leçons pour aujourd'hui) appelle «massacrer et s'en aller» : la tendance des régimes colonisateurs à pacifier ou punir, puis passer leur chemin et faire un travail médiocre en ce qui concerne l'établissement d'une administration quotidienne. Ce pourrait bien être — c'est du reste l'inquiétude de Ferguson - ce que le gouvernement de George Bush a en tête ${ }^{19}$. En Irak, cependant, «s'en aller » se révèle la tâche la plus difficile.

Il existe un autre modèle de colonisation censée être bienveillante, plus cohérent avec la vision qu'un gouvernement conservateur se fait du monde : une vue plus minimaliste de la façon dont on maintient la paix coloniale dans laquelle des peuples divisés et primitifs sont tenus à l'écart des menaces des autres et se voient donner la chance de développer une agriculture et un commerce plus productifs, sous un contrôle administratif et judiciaire qui, à défaut d'être équitable, est prévisible ${ }^{20}$. 
La familiarité avec le passé doit encore conduire au scepticisme à propos de ce futur. La colonisation de l'Afrique a éteint certaines formes de conflits - on ne doit pas se forger l'image romantique d'une Afrique pacifique pour mettre en question l'idée d'une paix coloniale. Mais, significativement, les racines des conflits africains actuels se trouvent dans l'ethnicisation que la stratégie coloniale de domination par les élites indigènes — gelées sur place par l'autorité colonisatrice — a favorisée, hors des modèles plus changeants de différence culturelle et des efforts des dominants pour recruter clients et fidèles. L'autorité de la loi était à peine un acquis colonial : cette notion était traversée par la ségrégation raciale en matière d'emploi, de résidence et de services publics, et par l'idée que les Africains devraient être dominés par la «coutume» et que leur terre ne pourrait être que « communautaire ». Quand, après la Deuxième Guerre mondiale, les gouvernements britannique et français essayèrent enfin de réduire les exclusions raciales qui faisaient partie de la vie quotidienne dans les colonies, avec l'exception notable des colonies de peuplement blanc, cela faisait partie de la modernisation ambitieuse de l'impérialisme, avec tous les coûts et conflits que cela entraînait. Comme un gouvernement américain qui n'est pas connu pour son programme réformateur sur le plan social semble le découvrir en Irak, établir l'autorité de la loi, la sécurité des transactions économiques et le respect intercommunautaire pourrait bien être un des processus les plus coûteux, les plus incertains et les plus longs qui soient. Seul l'aveuglement de certains conservateurs face à la complexité de la vie sociale et leur ignorance de l'histoire conflictuelle des empires du $\mathrm{xx}^{\mathrm{e}}$ siècle rendent possible leur vision de l'occupation coloniale comme un précédent pour établir légalité et transparence dans l'administration.

En fin de compte, l'argument de Ferguson pour la transmission du manteau impérial de la Grande-Bretagne aux États-Unis ne porte nullement sur l'empire. La masse de son livre Empire est assez scrupuleuse pour distinguer l' « empire » comme forme politique de l'autorité de la loi, de l'honnêteté, du souci des autres et de la généralisation des bénéfices du développement économique. Dans ses pages de conclusion, ces vertus sont liées non pas à l'histoire désordonnée et souvent sordide de l'empire, mais à l'image d'un homme britannique portant le « fardeau de l'homme blanc » et sauvant les peuples qui ne peuvent se sauver eux-mêmes ${ }^{21}$.

Mais le bref épisode de la modernisation de l'impérialisme britannique et français des années 1950 mérite encore qu’on y réfléchisse, non pas 
tant pour ce qu'il fut que pour ses conséquences inattendues : l'augmentation des revendications exprimées dans le langage de l'impérialisme d'après-guerre. Cette histoire rappelle que les termes de la politique ne sont pas statiques, et que les gouvernements qui prennent un pouvoir mondial et s'immiscent profondément dans les affaires d'un autre État connaîtront sans doute des répercussions bien au-delà de l'objectif visé. Nous devons penser précisément aux spécificités des institutions et aux possibilités de différents langages par lesquels les mouvements politiques formulent des revendications et remettent en cause l'ordre établi. Nous ne sommes pas contraints à choisir entre la politique des États-nations et de la citoyenneté ou une globalité amorphe, non plus qu'entre d'inviolables souverainetés et des ingérences effrénées. Plus tôt nous commencerons à penser de façon soigneuse et précise l'espace qui se trouve entre ces grands choix, et mieux ce sera.

\section{Traduit de l'anglais (États-Unis) par Pierre Savy}

1. Le basculement est incarné dans les arguments contraires de deux livres portant le titre d'Empire : l'un, écrit par Michael Hardt et Antonio Negri (Paris, Exils, 2000), dissout la politique dans des réseaux globaux amorphes mais tout-puissants ; l'autre, par Niall Ferguson (Londres, Allen Lane, 2003), se conclut sur un plaidoyer pour que les États-Unis revêtent le manteau de l'Empire britannique — argument qui contredit sa conception relativement critique, parfois cynique, de l'empire tel qu'il a réellement existé. 2. Comme Craig Calhoun l'a fait valoir, le sentiment de communauté vient de ce que l'on pense et de ce que l'on débatte des constitutions, des institutions et des normes — et pas seulement de sentiments antérieurs ; cela rend plus important l'effort pour poser des questions sur la souveraineté partagée et compromise et sur les institutions non nationales. « Imagining Solidarity : Cosmopolitanism, Constitutional Patriotism, and the Public Sphere », Public Culture, 14, 2002, p. 147-171.

3. Dans les communications qu'ils ont présentées au séminaire «Lessons of Empire » [New York University, 26-27 septembre 2003, URL : http://conconflicts.ssrc.org/lessonsofempire/ (visité le 19 mai 2010)], George Steinmetz et Jack Snyder ont proposé de distinguer entre différents modes d'intervention de la part d'un gouvernement puissant dans les affaires de gouvernement plus faibles : le mode impérial (l'intervention dans un autre gouvernement sans le gouverner vraiment), le mode hégémonique (fixer les règles du jeu que les autres doivent suivre) et le mode colonial (gouverner les affaires intérieures d'un gouvernement subordonné). Ces catégories n'épuisent pas toutes les possibilités, et historiquement la catégorie d' « empire » inclut une gamme considérable de façons dont l'incorporation de différents groupes de population et la différenciation de ces groupes furent opérées et contestées.

4. Donald Hepburn, « Nice War. Here's the Bill », New York Times, 3 septembre 2003, A19. L'auteur n'est pas un universitaire de gauche ou un professionnel du développement, mais un cadre de l'industrie pétrolière.

5. Cet article s'inspire de travaux déjà publiés. Pour la question générale des ambiguïtés de la domination coloniale, voir Frederick Cooper et Ann Stoler, « Between Metropole and Colony : Rethinking a Research Agenda », dans id. (dir.), Tensions of Empire : Colonial Cultures in a Bourgeois World, 


\section{La modernisation du colonialisme et les limites de l'empire}

Berkeley, University of California Press, 1997, p. 1-56, et Frederick Cooper, Le Colonialisme en question. Théorie, connaissance, histoire, Paris, Payot-Rivages, 2010 [2005]. Pour la question du dernier épisode, avorté, de modernisation coloniale, voir Frederick Cooper, Décolonisation et travail en Afrique. L'Afrique britannique et française, 1935-1960, Paris, Karthala, 2004 [1996].

6. Alice Conklin, A Mission to Civilize : The Republican Idea of Empire in France and West Africa, 1895-1930, Stanford, Stanford University Press, 1998.

7. Thomas Holt, The Problem of Freedom : Race, Labor and Politics in Jamaica and Britain, 1832 1938, Baltimore, Johns Hopkins University Press, 1990 ; Catherine Hall, Civilising Subjects : Metropole and Colony in the English Imagination, 1830-1867, Chicago, University of Chicago Press, 2002.

8. D'où la grande préoccupation des défenseurs du nouvel impérialisme : que cela soit fait de façon juste, que ses coûts considérables soient reconnus et que le nouveau pouvoir impérial agisse conformément aux règles du droit et à l'opinion internationale. Sur tous ces points, les motifs de doute ne manquent pas, ainsi que l'a reconnu Michael Ignatieff, «The American Empire : The Burden », The New York Times Magazine, 5 janvier 2003.

9. Seeley cité par Dominic Lieven, Empire : The Russian Empire and Its Rivals, New Haven, Yale University Press, 2001, p. 108. Sur l'idée que la Grande-Bretagne était un « titan prudent », réticent à tout effort pour organiser l'ordre international, plutôt qu'un « titan fatigué » — une puissance anciennement hégémonique qui perdit ensuite l'initiative — , voir John M. Hobson, « Two Hegemonies or One ?A Historical-Sociological Critique of Hegemonic Stability Theory », dans Patrick Karl O'Brien et Armand Clesse (dir.), Two Hegemonies : Britain 1846-1914 and the United States 1941-2001, Londres, Ashgate, 2003, p. 316.

10. N. Ferguson, Empire, op. cit. Comparer les conclusions, p. 357-370, avec le noyau du livre. Pour l'idée de l'exercice de la puissance coloniale à travers les « despotismes décentralisés » de chefs africains sous autorité britannique, voir Mahmood Mamdani, Citoyen et sujet. L'Afrique contemporaine et l'héritage du colonialisme tardif, Paris, Karthala, 2004 [1996]. Mamdani démontre de façon convaincante que l'intention de la Grande-Bretagne, surtout dans les années 1920 et 1930, était de régner par l'intermédiaire d'entités « tribales », mais il n'explore pas les liens transversaux que des Africains nouèrent non plus que la mesure dans laquelle la mobilisation politique dans les années 1940 et 1950 développa des alternatives viables, pour un certain temps en tout cas. Sur la loi, voir Martin Chanock, Law, Custom and Social Order : The Colonial Experience in Malawi and Zambia, Cambridge, Cambridge University Press, 1985.

11. Stephen Constantine, The Making of British Colonial Development Policy 1914-1940, Londres, Cass, 1984.

12. S. Herbert Frankel, Capital Investment in Africa ; its Course and Effects, Londres, Oxford University Press, 1938. Sur l'ambivalence du capital français à propos de l'investissement colonial, voir Jacques Marseille, Empire colonial et capitalisme français : histoire d'un divorce, Paris, Albin Michel, 1984.

13. Sur les limites du développement capitaliste dans l'Afrique rurale, voir Sara Berry, No Condition is Permanent : The Social Dynamics of Agrarian Change in Sub-Saharan Africa, Madison, University of Wisconsin Press, 1993.

14. Pierre-Henri Teitgen, Assemblée nationale, Débats, 20 mars 1956, p. 1072-1073.

15. Cette idée est aussi présente chez Wm. Roger Louis et Ronald Robinson, « The Imperialism of Decolonization », Journal of Imperial and Commonwealth History, 22, 1994.

16. Une influente série d'articles publiés par un journaliste conservateur dans la presse parisienne mena à une discussion publique sur la question de savoir si les colonies africaines payaient, à une époque où le ministre d'Outre-mer produisait des rapports ambigus sur les résultats du processus de développement. En Grande-Bretagne, le Premier Ministre Macmillan, peu après Suez, commanda une analyse des coûts et des bénéfices colonie par colonie, dont les résultats ne furent pas particulièrement encourageants. Voir F. Cooper, Décolonisation et travail, op. cit., chapitre 8.

17. Un cas fameux de croissance économique menant à la révolte est celui des Mau Mau, qui éclata parmi les Kikuyu du Kenya en 1952. Il faisait suite non seulement à l'intensification de l'agriculture dans les fermes des colons blancs — d'où l'expulsion de beaucoup d'occupants illégaux kikuyu qui 


\section{Labyrinthe, $n^{\circ} 35$}

combinaient travail pour les blancs et travail en propre comme fermiers - mais aussi au développement accéléré d'une agriculture commerciale par les Kikuyu les plus aisés (à la faveur de la levée des restrictions raciales sur la culture du café), soucieux de ne laisser aucun occupant illégal expulsé de son village y obtenir des terres. L'épisode des Mau Mau fut, à plus d'un titre, une crise d'intensification $\mathrm{du}$ « développement ». Voir John Lonsdale et Bruce Berman, Unhappy Valley : Conflict in Kenya and Africa, livre 2, Violence and Ethnicity, Londres, James Currey, 1992 ; Tabitha Kanogo, Squatters and the Roots of Mau Mau, 1905- 63, Londres, James Currey, 1987.

18. Pour une interprétation de la révolution algérienne qui met à mal le mythe d'une lutte armée victorieuse et insiste sur le rôle des facteurs internationaux dans son règlement, voir Matthew Connelly, A Diplomatic Revolution: Algeria's Fight for Independence and the Origins of the Post-Cold War Era, New York, Oxford University Press, 2002. Plus généralement, j'ai écrit sur la relation entre luttes dans les empires et luttes contre les empires dans « Mobilization and Accommodation », conférence à l'Institut des hautes études internationales, Genève, mars 2003.

19. Niall Ferguson, « The Empire Slinks Back : Why Americans Don't Really Have What It Takes to Rule the World », The New York Times Magazine, 27 avril 2003, p. 52-57. L'expression « massacrer et s'en aller » vient d'Empire, p. 179.

20. Deepak Lal développe l'argument tortueux d'un impérialisme américain qui détacherait la domination minimaliste d'un programme réformateur ou moralisateur. Cela ne permet pas de surmonter la difficulté qu'il y a à séparer ce qui fait la domination minimaliste du bagage idéologique porté par les institutions légales et administratives. Et cela revient à une interprétation fautive du gouvernement américain, qui semble souvent avoir pour objectif premier le maintien de sa forteresse chrétienne et qui montre peu d'intérêt à rendre des comptes à d'autres qu'à lui-même. Deepak Lal, « In Defense of Empires », discours à l'American Enterprise Institute, 30 octobre 2002, www.econ.ucla.edu/Lal/papers/In defense of empires.pdf (visité le 19 mai 2010).

21. L'évocation par Ferguson de ce texte trop utilisé et trop cité de Kipling est dans Empire, p. 369. 